\title{
The assembly of the adult skeleton during growth and maturation: implications for senile osteoporosis
}

\author{
B. Lawrence Riggs, ${ }^{1}$ Sundeep Khosla, ${ }^{1}$ and L. Joseph Melton III $^{2}$ \\ ${ }^{1}$ Endocrine Research Unit, and \\ ${ }^{2}$ Department of Health Sciences Research, Mayo Clinic and Foundation, Rochester, Minnesota 55905, USA
}

Address correspondence to: B.L. Riggs, Endocrine Research Unit, 5-164 West Joseph Building, St. Mary's Hospital, Rochester, Minnesota 55905, USA. Phone: (507) 284-3961; Fax: (507) 284-8271: E-mail: riggs.lawrence@ mayo.edu.

Senile osteoporosis is a pediatric disease.

-Charles E. Dent

Almost 30 years ago, the late Professor Charles Dent of University College, London pointed out that failure to gain sufficient bone during skeletal growth and maturation predisposes to the development of senile osteoporosis as age-related bone loss ensues. While, much more is known about the patterns of bone loss than about patterns of bone growth, the following is agreed upon. Between the onset of puberty and young adulthood, skeletal mass doubles (1). Most of this increase occurs during the pubertal growth spurt, although small increases in bone mineral density (BMD) continue for an undefined interval after linear growth ceases, by a process termed bone consolidation. The increases in statural height and bone remodeling are greatest in early puberty and then progressively decline until the epiphyses close in late puberty (1-5). In contrast, maximal increases in BMD occur 2 years later - at menarche in girls and in late puberty in boys. The pattern of bone growth in boys differs from that in girls in 2 ways. First, boys have 2 more years of prepubertal growth because of a later onset of puberty (age 14, rather than 12 as in girls). Second, their pubertal growth spurt lasts for 4 years rather than the 3 years in girls $(2,3,5)$. These differences largely account for the greater peak bone mass in boys.

With a few exceptions, previous studies have focused on the overall pattern of skeletal growth and maturation. However, in this issue of the JCI, Bass et al. (6) report on a comprehensive, longitudinal study of skeletal changes across puberty in 109 girls and describe specific differences in growth changes by bone types, bone regions, and bone surfaces. Both bone size and volumetric BMD
(vBMD) were assessed, which is particularly important for studies of skeletal growth in which both are changing. It is not always appreciated that dual-energy $\mathrm{x}$-ray absorptiometry, the method used for more than $90 \%$ of bone densitometry measurements worldwide, assesses areal BMD (aBMD), measured in $\mathrm{g} / \mathrm{cm}^{2}$, rather than vBMD, measured in $\mathrm{g} / \mathrm{cm}^{3}$. Unfortunately, aBMD overestimates vBMD substantially by failing to account for wider bones also being deeper. Bass et al. assessed vBMD indirectly by making geometrical conversions from aBMD. They find that growth within the skeleton is not uniform during sexual maturation. Thus, prepubertal growth is disproportionately greater in the legs, whereas the adolescent growth spurt is disproportionately greater in the trunk. In both the appendicular and the axial skeleton, Bass et al. and others $(1,3,4)$ find that most of the increase in bone mass in early puberty is due to increases in bone size, whereas a smaller increase in vBMD that is independent of increases in bone size occurs in late puberty. This distinction cannot be made with measurements of aBMD. Moreover, in the cortical bone of the femurs and metacarpals, Bass et al. find different patterns of growth on bone surfaces. Both the periosteal and endocortical diameters increase during puberty (the former because of a relative excess of bone apposition, and the latter because of a relative excess of bone resorption), but the rate of expansion is more at the periosteum, leading to a progressive increase in cortical width. Interestingly, in late puberty (between 1.0 and 3.7 years after menarche), rather than continued expansion, there is contraction in endocortical diameter, indicating that apposition now predominates over resorption at the endocortical surface. This leads to an even greater increase in cortical width. Also, there was a sharp but transient increase in true vBMD (defined as bone mineral content within bone itself, excluding the marrow cavity) during the first year after menarche.

What are the main hormonal determinants of these skeletal changes during puberty? Bass et al. find that serum IGF-1 levels increase by over 2 -fold from puberty to menarche - the period of rapid skeletal growth - and then remain relatively constant until linear growth is terminated by epiphyseal plate closure. Their data, along with those of others $(5,7)$, suggest that an increase in serum IGF-1, stimulated by growth hormone (7) and sex steroids (8), is the major facilitator of increases in bone size during puberty. Also, serum estradiol correlated inversely with biochemical markers for bone turnover, consistent with the antiresorptive action of estrogen. Thus, the surge in the production of estrogen at menarche that closes the epiphyses is also the likely cause of the temporally associated increases in vBMD and cortical true $v B M D$, and of the conversion from endochondrial surface expansion (increasing diameter) to contraction (decreasing diameter) in cortical bone.

On the basis of these observations, Bass et al. hypothesize that childhood diseases that induce growth arrest will have different skeletal consequences depending on the age of exposure. In prepuberty, they will affect mainly the limbs; in early puberty, mainly the vertebrae; and in late puberty, mainly the enhancement in vBMD. This interesting theoretical concept warrants experimental testing. Bass et al. also firmly 
conclude that osteoporosis of old age may have its foundation in growth. Although the importance of peak bone mass seems indisputable, it is important to acknowledge the equally important role of bone loss in determining residual bone mass late in life in both genders, but particularly in postmenopausal women (9-11). The original hypothesis of Newton-John and Morgan (12), that age-related bone loss occurs at a relatively constant rate among individuals, is no longer generally accepted. In fact, bone loss has been shown to be highly variable over life (11, 13), and differences in individual rates probably account for up to half of the variance of cancellous bone mass (14, 15) and about one-third of the variance in cortical bone mass $(10,11)$ late in life. Thus, a comprehensive description of pathogenesis should encompass the contributions of both low peak bone mass and rapid bone loss, and should recognize that for a given patient, either or both of these processes may be the principal cause of osteoporosis.

1. Katzman, D.K., Bachrach, L.K., Carter, D.R., and Marcus, R. 1991. Clinical and anthropometric correlates of bone mineral acquisition in healthy adolescent girls. J. Clin. Endocrinol. Metab. 73:1332-1339.

2. Theintz, G., et al. 1992. Longitudinal monitoring of bone mass accumulation in healthy adolescents: evidence for a marked reduction after 16 years of age at the levels of lumbar spine and femoral neck in female subjects. J. Clin. Endocrinol. Metab. 75:1060-1065.

3. Lu, P.W., Cowell, C.T., Lloyd-Jones, S.A., Briody, J.N., and Howman-Giles, R. 1996. Volumetric bone mineral density in normal subjects, aged 5-27 years. J. Clin. Endocrinol. Metab. 81:1586-1590.

4. Gilsanz, V., et al. 1998. Differential effect of race on the axial and appendicular skeletons of children. $J$. Clin. Endocrinol. Metab. 83:1420-1427.

5. Cadogan, J., Blumsohn, A., Barker, M.E., and Eastell, R. 1998. A longitudinal study of bone gain I pubertal girls: anthropometric and biochemical correlates. J. Bone Miner. Res. 13:1602-1612.

6. Bass, S., et al. 1999. The differing tempo of growth in bone size, mass, and density in girls is regionspecific J.Clin. Invest. 104:795-804.

7. Martha, P.M., Jr., and Reiter, E.O. 1991. Pubertal growth and growth hormone secretion. Endocrinol.
Metab. Clin. North Am. 20:165-182.

8. Kerrigan, J.R., and Rogol, A.D. 1992. The impact of gonadal steroid hormone action on growth hormone secretion during childhood and adolescence. Endocr. Rev. 13:281-298.

9. Riggs, B.L., Khosla, S., and Melton, L.J., III. 1998. A unitary model for involutional osteoporosis: estrogen deficiency causes both type I and type II osteoporosis in postmenopausal women and contributes to bone loss in aging men. J. Bone Miner. Res. 13:763-773.

10. Riggs, B.L., et al. 1982. Changes in bone mineral density of the proximal femur and spine with aging: differences between the postmenopausal and senile osteoporosis syndromes. J. Clin. Invest. 70:716-723.

11. Hansen, M.A., Overgaard, K., Riis, B.J., and Christiansen, C. 1991. Role of peak bone mass and bone loss in postmenopausal osteoporosis: 12 year study. BMJ. 303:961-964.

12. Newton-John, H.F., and Morgan, D.B. 1968. Osteoporosis: disease or senescence? Lancet. 1:232-233.

13. Laib, A., Hauselmann, H.J., and Ruegsegger, P. 1998. In vivo high resolution 3D-QCT of the human forearm. Technol. Health Care. 6:329-337.

14. Block, J.E., et al. 1989. Models of spinal trabecular bone loss as determined by quantitative computed tomography. J. Bone Miner. Res. 4:249-257.

15. Meier, D.E., Orwoll, E.S., and Jones, J.M. 1984 Marked disparity between trabecular and cortical bone loss with age in healthy men. Ann. Intern. Med. 101:605-612. 